\title{
ABORDAGEM DA EROSÃO DENTÁRIA NA CLÍNICA ODONTOPEDIÁTRICA: RELATO DE CASOS
}

\section{APPROACH OF DENTAL EROSION IN PEDIATRIC DENTISTRY CLINIC: CASE REPORTS}

\author{
Camila Leite Fontes* \\ Luanda Cristina de Oliveira Luciano* \\ Meire Coelho Ferreira"* \\ Marco Aurélio Benini Paschoal ${ }^{* * *}$
}

\begin{abstract}
RESUMO
A erosão dentária em crianças e adolescentes está cada vez mais presente devido a mudanças no estilo de vida das pessoas, havendo um crescente aumento de sua prevalência nos dias atuais. Sendo assim, o objetivo deste trabalho foi relatar três casos clínicos de pacientes infantis com presença de erosão dentária associada ao consumo excessivo de bebidas ácidas. Para os pacientes e pais/responsáveis foi fornecido um questionário contendo perguntas relacionadas à condição estudada. Para todos os pacientes, realizou-se limpeza dos dentes com escova de Robson e pasta profilática. Após secagem, os elementos dentários foram submetidos à identificação dos sinais clínicos da erosão e classificados quanto à severidade, usando-se o escore de BEWE. Os pacientes foram identificados com graus 1 e 2 de erosão dentária. Após essa identificação, foram submetidos a aplicação semanal de verniz de flúor durante quatro semanas consecutivas. O questionário aplicado demonstrou que os pacientes faziam uso frequente de refrigerantes do tipo cola e suco de frutas como laranja, maracujá e acerola. Os pacientes, assim como os responsáveis, foram orientados quanto à erosão dentária e adoção de medidas para o impedimento de sua progressão. Conclui-se que é fundamental esclarecer os pacientes sobre as causas e consequências do desgaste erosivo, visando a preservação da estrutura dentária ainda em estágio precoce.
\end{abstract}

Descritores: Erosão dentária; Odontopediatria; Diagnóstico; Tratamento

\section{ABSTRACT}

Nowadays, dental erosion in children and teenagers is more present due to changes in life style, which increases the prevalence of that condition. Hence, the aim of the present study was to report three clinical cases of pediatric patients with presence of dental erosion associated with the high consumption of acid beverages. For each patient and guardians was provided a questionnaire containing questions related to the studied condition. For all patients, was held teeth cleaning with Robson brush and prophylaxis paste. After drying, the dental elements were subjected to identification of clinical signs of erosion and classified according to severity using the score of BEWE. After this identification, the patients underwent weekly application of fluoride varnish for four consecutive weeks. The patients were identified with 1 and 2 degrees of dental erosion. The questionnaire showed that they made frequent use of soft drinks type cola based and fruit juices like orange, passion fruit and acerola. Patients, as well as their responsibles, were oriented to the dental erosion and preventing the progression of the presented condition. It is mandatory to clarify and explain to patients and guardians about the dental erosion causes and its consequences of erosive wear aiming to preserve the dental structure in the early stages. Descriptors: Tooth erosion; Pediatric Dentistry; Diagnosis; Treatment

* Universidade CEUMA - São Luís/MA. Graduanda em Odontologia. camilafontes5@hotmail.com2 Universidade CEUMA - São Luís/MA. Especialista em Dentística pela Faculdade Ingá - Imperatriz/MA e Mestranda do curso de Pós-Graduação do Programa de Odontologia Integrada luanda_cris@ hotmail. com

** Universidade CEUMA - São Luís/MA. Especialista em Dentística pela Faculdade Ingá - Imperatriz/MA e Mestranda do curso de Pós-Graduação do Programa de Odontologia Integrada luanda_cris@hotmail.com

*** Universidade CEUMA - São Luís/MA. Especialização em Odontopediatria pela Univale, Mestrado em Odontologia pela Universidade Federal de Santa Catarina UFSC, Doutorado em Odontologia pela UFSC e Pós-Doutorado em Epidemiologia pela Universidade Federal dos vales do Jequitinhonha e Mucuri, Professora do curso de Pós-Graduação do Programa de Odontologia Integrada da Universidade CEUMA-São Luís/MA meirecofe@hotmail.com

***** Universidade CEUMA - São Luís/MA. Mestre em Ciências Odontológicas aplicadas pela FOB/USP. Doutor em Ciências Odontológicas pela FOAr- UNESP e Pós-Doutorado na NYU - NY. Professor do curso de Pós-Graduação do Programa de Odontologia Integrada da Universidade CEUMA. marcobpaschoal@hotmail.com 


\section{N T RO DUÇÃO}

O aumento da prevalência da erosão dentária em crianças e adolescentes está diretamente relacionado às mudanças no estilo de vida, sendo tal condição evidenciada por estudos epidemiológicos que mostram crianças em fase de dentição decídua e mista com maior predisposição para o desenvolvimento da lesão ${ }^{1}$.

A erosão dentária é definida como uma perda progressiva e irreversível de tecido dentário, incialmente ocasionando a desmineralização do esmalte por um processo químico sem o envolvimento de microrganismos. $\mathrm{O}$ principal fator etiológico é a presença de ácidos na cavidade bucal, podendo ser classificados como de origem extrínseca ou intrínseca. Enquanto as fontes extrínsecas estão relacionadas a hábitos alimentares e estilo de vida, as causas intrínsecas podem ser provocadas por doenças sistêmicas².

Em relação a fatores extrínsecos, houve um maior consumo de bebidas com baixo pH (ex. sucos prontos e refrigerantes) por crianças na fase escolar. O Ministério da Saúde evidenciou um alto consumo de refrigerantes entre crianças de 9 a 12 meses de vida ${ }^{3}$. Quanto aos fatores intrínsecos, podem ser citados os transtornos alimentares como a anorexia e a bulimia. Adicionalmente, estresse diário e altos níveis de ansiedade podem levar ao desenvolvimento de doenças gastrointestinais, e assim, essas emoções podem proporcionar vômitos, regurgitações e refluxos que levam a um contato direto do ácido gástrico com a superfície dentária, causando lesões erosivas ${ }^{4}$.

Para se determinar a erosão dentária é preciso analisar alguns fatores, tais como o pH do meio bucal, capacidade tampão da saliva, a concentração de cálcio e fosfato, além da presença de fluoretos. Ainda, a frequência, a quantidade e a forma como essas bebidas são ingeridas são outros fatores determinantes ${ }^{5}$. Clinicamente, o esmalte apresenta-se com diminuição da lisura e brilho, uma fina camada de esmalte com aspecto amarelado e halo translúcido, bordas incisais finas ou fraturadas, concavidades dentinárias bem definidas, comprometendo a estética e a função mastigatória e, em casos mais graves, há perda da dimensão vertical podendo ocorrer exposição pulpar e resultar em sensibilidade e dor. ${ }^{6}$

Como forma de tratamento, o primeiro passo é a eliminação do efeito causal, no intuito de prevenir a evolução e o aparecimento de novas lesões ${ }^{7}$. O cirurgião-dentista tem o papel de reconhecer, diagnosticar, orientar, tratar e acompanhar regularmente a lesão, a fim de promover uma estabilização desse desgaste que pode acontecer de forma rápida e progressiva $^{2}$. Dependendo do grau de desgaste, os tipos de tratamento serão indicados desde aplicações de verniz fluoretado a procedimentos restauradores convencionais, diretos ou indiretos ${ }^{2}$ acompanhados de mudança de hábitos comportamentais do indivíduo. Sendo assim, o presente trabalho teve como objetivo relatar casos clínicos de três pacientes infantis com erosão dentária e que apresentavam alto consumo de bebidas ácidas.

\section{RELATOS DOS CASOS}

Paciente C. S. C., do gênero feminino, de nove anos de idade, foi atendida na clínica de Odontopediatria da Universidade Ceuma, São Luís-MA, devido a queixa de sensibilidade quando da ingestão de bebidas frias e quentes. Uma segunda paciente, R. O. R., gênero feminino onze anos de idade, ao exame inicial, foram verificadas lesões de desgaste dentário, similarmente como no caso do paciente G. S. M., gênero masculino, cinco anos de idade, que, ao ser submetido a tratamento odontológico, verificaram-se desgastes em esmalte e dentina. Dessa forma, os pais/responsáveis legais dos pacientes,
FONTES CL

LUCIANO COL

FERREIRA MC

PASCHOAL MAB

ABORDAGEM DA EROSÃO DENTÁRIA NA CLÍNICA ODONTOPEDIÁTRICA: RELATO DE CASOS

263
REV, OdONTOL.

UNIV, CID, SÃO PAULO

$2016 ; 28(3): 262-$

9 , SET-DEZ 
FONTES CL LUCIANO COL FERREIRA MC

PASCHOAL MAB

ABORDAGEM DA EROSÃO DENTÁRIA

NA CLINICA ODONTOPEDIÁTRICA: RELATO DE CASOS

após assinatura do Termo de Consentimento Livre e Esclarecido (TCLE), autorizaram a anamnese e o tratamento odontológico e foram submetidos a um questionário, de modo a serem investigas as queixas dos achados clínicos (CEP Proc. 1.180.381).

Inicialmente, para todos os pacientes, realizou-se limpeza dos dentes com escova de Robson e pasta profilática. Após secagem, os elementos dentários foram submetidos à identificação dos sinais clínicos da erosão e classificados quanto à severidade, usando-se o Escore BEWE (Basic Erosive Wear Examination), o qual tem por objetivo o diagnóstico e conduta de tratamento de lesões erosivas. ${ }^{7}$

Constataram-se lesões de erosão classificadas em Escore 1 (perda inicial da textura da superfície) nos incisivos

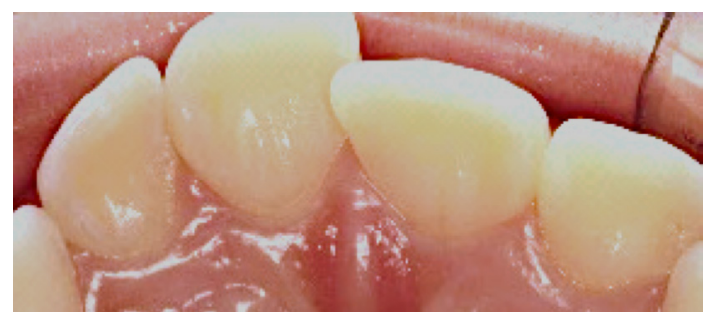

Figura 1. Lesões erosivas nos incisivos permanentes.

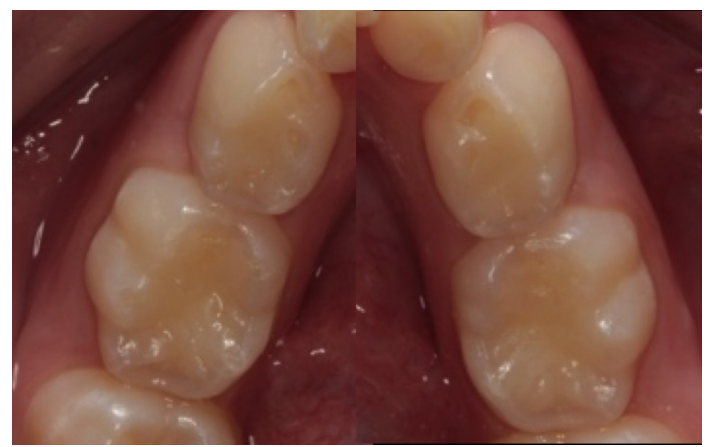

Figuras 2. Lesões erosivas em molares decíduos inferiores.

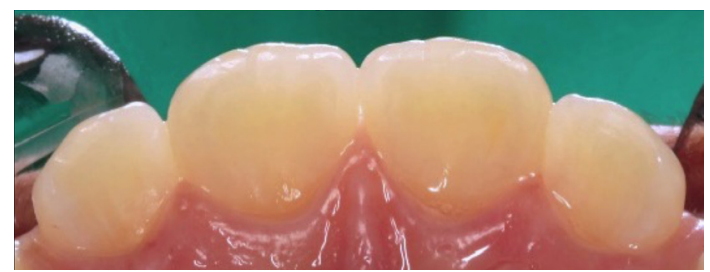

Figura 3. Lesões erosivas em incisivos permanentes superiores.

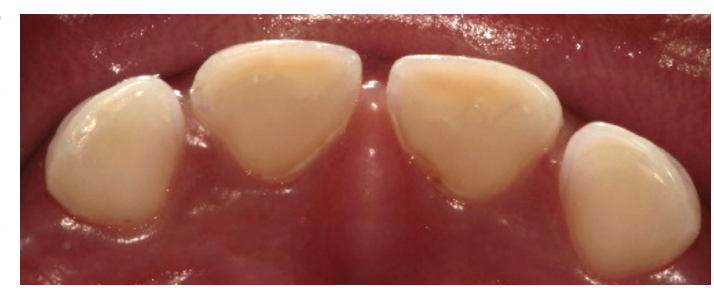

Figura 4. Lesões erosivas em incisivos decíduos superiores.

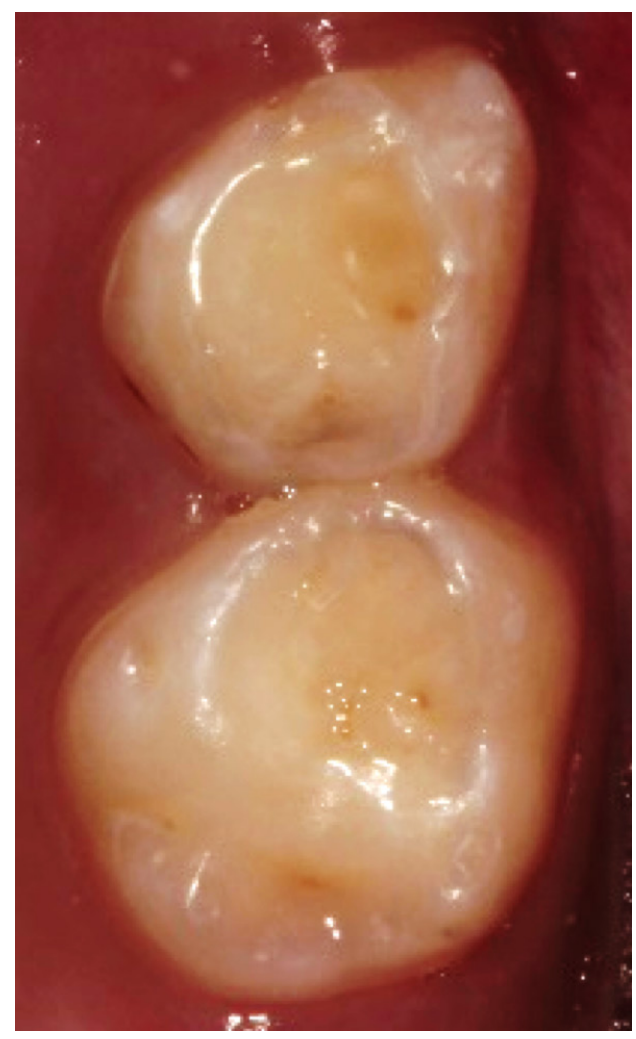

Figura 5. Lesões erosivas em molares decíduos superiores.

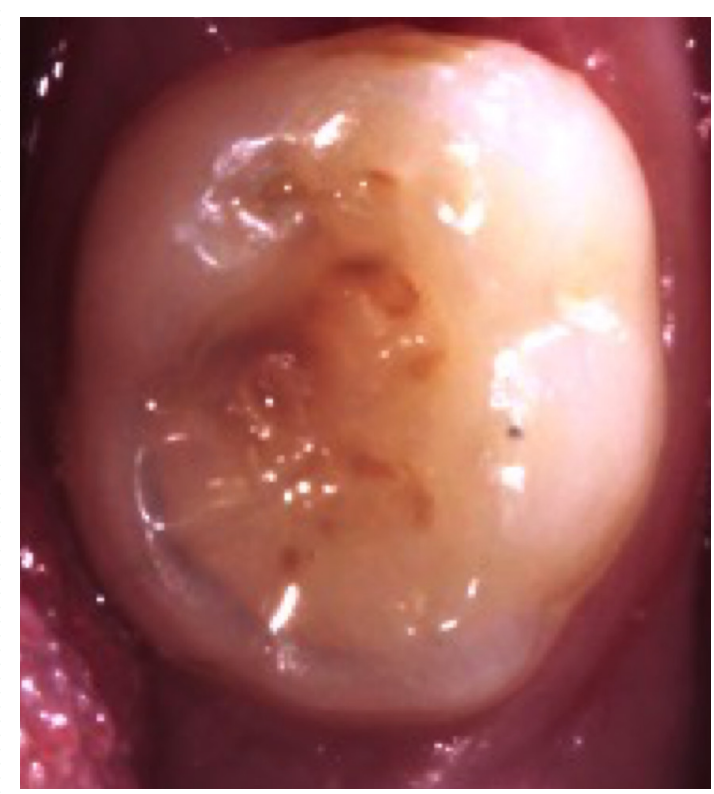

Figura 6. Lesões erosivas em molar decíduo inferior. 
permanentes (Figura 3) e molares decíduos superiores e inferiores no primeiro caso. Já no segundo caso, a paciente foi diagnosticada também com escore 1, entretanto apenas nos incisivos permanentes (Figura 2). No último caso, foram observados os escores 1 e 2 (presença de um defeito distinto com perda de tecido duro menor que $50 \%$ da superfície), quadro este em que o tecido dentinário dos incisivos superiores e molares decíduos já se apresentavam afetados (Figuras 4, 5 e 6).

Após o diagnóstico da condição erosiva, foi aplicado um questionário aos pais/ responsáveis contento perguntas de cunho objetivo relacionadas à erosão dentária, a fim de se investigar a causa e, dessa forma, a conduta mais aceitável.

Concluiu-se, a partir dos dados colhidos, que a etiologia das lesões erosivas desses casos estava relacionada principalmente com hábitos alimentares. $\mathrm{O}$ questionário demonstrou que os pacientes faziam uso frequente de refrigerantes do tipo cola e suco de frutas como laranja, maracujá e acerola, e todos se queixavam de sensibilidade dentária, sendo que um paciente já teria apresentado refluxo gastroesofágico, o que contribui diretamente para a piora do quadro erosivo. Após identificação das causas da erosão dentaria, os pacientes foram submetidos ao tratamento da condição encontrada.

Após secagem dos elementos dentários e isolamento relativo com rolos de algodão, os pacientes foram submetidos a aplicação semanal de verniz de fluoreto de sódio a 5\% (22.600 ppm de flúor, Duraphat, Colgate Palmolive Company $\AA$, New York, NY, EUA) com auxílio de um microbrush durante quatro semanas consecutivas. Os pacientes, assim como os responsáveis, foram orientados a mudanças dos hábitos prejudiciais, quanto a dieta para evitar o consumo de alimentos e bebidas ácidas, evitar o bochecho e a utilização de canudos; quanto à higiene, orientou-se aguardar o período de uma hora após a refeição para realizar a escovação, pois o esmalte aparenta um aspecto "macio" após o contato com a substância ácida e pode desgastar-se facilmente, utilização de escovas macias e pastas de dente com baixa abrasividade, redução da força de escovação; além disso, orientação quanto a visitas regulares ao dentista, pois o diagnóstico precoce possibilita a atuação do profissional para o impedimento da progressão do quadro erosivo tanto na dentição decídua como na permanente.

\section{DISCUSSÃO}

A alta prevalência de erosão dentária tem como um dos fatores desencadeantes principais o aumento da ingestão de bebidas e comidas ácidas, segundo pesquisa realizada com objetivo de avaliar a prevalência e os fatores etiológicos envolvidos com o desgaste dos dentes decíduos. Em uma amostra de conveniência foram selecionadas 356 crianças com idade de 6 anos de idade em Bauru, SP, quando se constatou que $34,8 \%$ dos incisivos, $64,75 \%$ dos caninos e $40,7 \%$ dos molares das crianças apresentavam desgaste do esmalte dentário classificado como grau $1 .{ }^{8}$

Os refrigerantes aparecem como um dos principais causadores de erosão dentária e, por isso, foi realizado um estudo com o objetivo de avaliar o efeito da frequência de ingestão de refrigerante na erosão de esmalte-dentina e investigar a capacidade biológica do papel da saliva na reversão das alterações. Os resultados mostraram que, em função da frequência de ingestão de refrigerante, a porcentagem de perda de dureza de blocos de esmalte e dentina foi de 18,7 a 27,9 e de 24,6 a 32,6 , respectivamente. A porcentagem de recuperação de dureza pela ação da saliva foi parcial, variando de 43,6 a 35,6 para o esmalte e de 40,5 a 34,6 para a dentina. Segundo a equipe, houve também uma correlação significativa entre a frequência de ingestão da bebida e a porcentagem de perda de dureza. Dessa forma, os pesquisadores concluíram que, dependendo da frequência de ingestão de refrigerante, há perdas proporcionais e irreversíveis da estrutura superficial dos tecidos dentários. $^{9}$

A prevalência do desgaste dentário erosivo tem aumentado, principalmente em crianças e adolescentes. De acordo com autores, a taxa de erosão dentária em crianças varia de $2 \%$ a $57 \%$ nos estudos
FONTES CL

LUCIANO COL

FERREIRA MC

PASCHOAL MAB

ABORDAGEM DA EROSÃo DENTÁRIA NA CLÍNICA ODONTOPEDIÁTRICA : RELATO DE CASOS

265

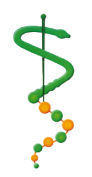

REV, ODONTOL.

UNIV, CID, SÃO

PAULO

2016; 28(3): 262 -

9, SET-DEZ 
FONTES CL

LUCIANO COL

FERREIRA MC

PASCHOAL MAB

ABORDAGEM DA EROSÃO DENTÁRIA

NA CLINICA

ODONTOPEDIÁTRICA:

RELATO DE CASOS

\section{$266 \ldots$}

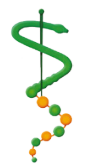

REV, ODONTOL.

UNIV, CID, SÃO

PAULO

$2016 ; 28(3): 262-$

9, SET-DEZ clínicos. ${ }^{10}$ Uma revisão sistemática recente indicou que a prevalência de desgaste envolvendo dentina variou em até $82 \%$ e $54 \%$ para dentes decíduos e dentes permanentes, respectivamente, em crianças de até 7 anos de idade. ${ }^{11}$ Corroborando com esses resultados, uma amostra aleatória de 1035 crianças de 14 anos de idade encontrou que $30 \%$ apresentavam dentina exposta na incisal e $8 \%$ exposição em superfícies oclusais e/ou lingual. ${ }^{12}$ Outro estudo realizado com 101 crianças de 4 a 16 anos de idade encontrou a exposição da dentina em $30 \%$ dos molares decíduos. ${ }^{13}$ Uma das principais razões pelas quais houve esse aumento, são as mudanças dos hábitos dietéticos da população, que tem consumido mais produtos industrializados, como os refrigerantes e bebidas isotônicas, o que pôde ser constatado pelos dados da pesquisa de orçamento familiar (POF) que apontou que, entre os anos de 1975 a 2003, a aquisição per capita de refrigerantes aumentou de 1,29 para 7,65 , o que equivale a $490 \%$ de crescimento. ${ }^{14}$

O diagnóstico precoce é de suma importância para que os danos erosivos sejam minimizados. Raramente os pacientes e/ou responsáveis conseguem reconhecer os sinais iniciais da erosão dentária, por isso, o profissional deve ficar atento aos sinais clínicos e conscientizar os pacientes de que o consumo de bebidas ácidas tem sido apontado como significante fator de risco à erosão dental. ${ }^{15}$

Nos casos clínicos relatados neste estudo, todos foram diagnosticados precocemente, devido à idade e porque a maioria apresentava lesões erosivas rasas em nível de esmalte, com exceção do terceiro caso, em que já se diagnosticou desgaste em nível dentinário, embora todos, sem exceção, apresentassem algum grau de sensibilidade dentária. Para todos os casos foi usado como diagnóstico o critério BEWE ${ }^{16}$ o qual é de fácil identificação e capaz de classificar de forma simples a erosão, por meio de escores que variam de 0 a $3 .{ }^{16}$ De forma similar, um estudo realizado teve como objetivo avaliar a prevalência, incidência, progressão e fatores associados à erosão dentária na dentição permanente de 1.528 adolescentes com 12 anos de idade, sendo os incisivos e primeiros molares permanentes examinados utilizando-se o mesmo critério do presente estudo. Como resultado, foram encontradas baixas taxas de prevalência e incidência de erosão dentária na população estudada, sendo classificadas com grau leve na grande maioria dos casos. ${ }^{17}$

No presente estudo foi aplicado um questionário capaz de identificar as principais causas da erosão, e foram investigados fatores que poderiam influenciar no desgaste dentário, como hábitos de higiene oral, força de escovação e saúde geral. Por meio do questionário, nos casos 1 e 2 foi encontrado histórico de indigestão, vômitos frequentes e refluxo, e todos relataram hábitos parafuncionais de roer unhas, colocar objetos na boca; em nenhum dos casos relataram fazer bochecho ou segurar líquido na boca antes de engolir; todos utilizam escovas com cerdas macias e aplicam força moderada durante a escovação. Fato expressivo foi o alto consumo diário de bebidas ácidas como refrigerantes do tipo à base de cola (Coca-Cola), à base de guaraná e laranja. Os sucos de frutas naturais e os industrializados mais consumidos eram os de sabor laranja, maracujá e acerola. Adicionalmente, os casos 1 e 2 relataram histórico de sensibilidade após a ingestão desses produtos.

Em relação às orientações e tratamento, foram aplicados os mesmos protocolos de outros dois autores em que, quanto ao primeiro, se basearam em orientações relativas ao consumo dietético e aos hábitos de higiene com instruções relativas ao tipo de escova, redução da força de escovação, além de aguardar de trinta minutos a uma hora após a refeição ácida para realizar a limpeza. Utilização de canudos, engolir rapidamente e evitar bochechos durante o consumo de bebidas ácidas também fizeram parte da orientação tanto aos pacientes quanto aos pais para supervisão de seus filhos. ${ }^{7,15}$

Como a modificação da dieta definitiva é uma tarefa árdua, é necessária a utilização de outras estratégias preventivas, entre elas, o uso de fluoretos. Quanto ao tratamento, foi preconizada a aplicação tópica de verniz fluoretado, pois tem sido considerado um dos melhores agentes 
pela presença de uma base de resina e sua substantividade. ${ }^{18} \mathrm{Em}$ função disso, o verniz se adere à superfície dentária, permitindo maior tempo de ação do fluoreto, agindo como uma barreira mecânica tanto para a prevenção como para a remineralização de lesões iniciais. ${ }^{19,20} \mathrm{O}$ flúor é o principal agente remineralizante indicado para prevenção e controle da erosão dental, agindo na redução da solubilidade da superfície, seguida do aumento da resistência superficial a partir da recuperação mineral. Nesse sentido, já se demonstrou que uma maior efetividade na proteção do substrato dental contra o desenvolvimento do desgaste erosivo está associada à aplicação de fontes de fluoreto com alta concentração. ${ }^{21}$

Em um estudo realizado no intuito de avaliar o efeito da solução de fluoreto de sódio ( $\mathrm{NaF}$ ) sobre a erosão inicial em esmalte, espécimes de esmalte preparados a partir de terceiros molares humanos foram tratados durante 24 horas com verniz Duraphat $(2,26 \% \mathrm{~F})$ ou 48 horas com solução de $\mathrm{NaF}(1,2 \% \mathrm{~F})$. Os espécimes foram lavados e imersos em bebida à base de cola (pH 2,6) durante 15 minutos, tendo como variável resposta a microdureza superficial logo após o tratamento com os dois diferentes protocolos e após 1,5 e 15 minutos. Os resultados demonstraram que ambos os tratamentos causaram aumento dos valores da dureza do esmalte e a subsequente inibição de amolecimento que foi estatisticamente significativa. ${ }^{22}$

Apesar de, na maioria, os dentifrícios utilizados atualmente serem fluoretados, a incidência mundial das lesões de erosão continua aumentando com o passar do tempo devido, principalmente, à modificação dos hábitos dietéticos, significando que, mesmo com a grande abrangência do flúor nas populações, existe uma tendência de aumento das lesões de erosão ao longo do tempo. ${ }^{23}$

\section{CONCLUSÃO}

Nos casos relatados, concluiu-se que é fundamental esclarecer aos pacientes sobre as causas e consequências do desgaste erosivo, visando a preservação da estrutura dentária ainda em estágio precoce. Espera-se que, por meio do tratamento aplicado junto com a conscientização e compreensão sobre a necessidade de mudanças no estilo de vida, se possa impedir a progressão das lesões erosivas encontradas, evitando-se o comprometimento da dentição permanente, e mantendo-se a saúde e integridade da estrutura dentária ao longo da vida.

\section{CONFLITO DE INTERESSES}

Os autores não têm qualquer conflito de interesse no presente estudo.
FONTES CL

LUCIANO COL

FERREIRA MC

PASCHOAL MAB

ABORDAGEM DA

EROSÃO DENTÁRIA

NA CLÍNICA

ODONTOPEDIÁTRICA:

RELATO DE CASOS

\section{7}

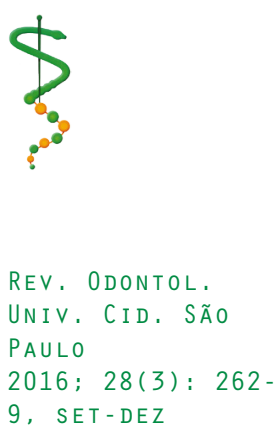


FONTES CL

LUCIANO COL

FERREIRA MC

PASCHOAL MAB

ABORDAGEM DA EROSÃO DENTÁRIA

NA CLINNICA

ODONTOPEDIÁTRICA:

RELATO DE CASOS

268
1. Lussi A, Jaeggi T. Erosion. diagnosis and risk factors. Clinical oral investigations. 2008;12 Suppl 1:S5-13.

2. Branco CA, Valdivia A, Soares $P$, Fonseca R, Fernandes Neto A, Soares C. Dental erosion: diagnosis and treatment options. Rev Odontol UNESP. 2008;37(3):235-42.

3. Brasil, Ministério da Saúde, Secretaria de Atenção à Saúde, Departamento de Ações Programáticas e Estratégicas. II pesquisa de prevalência de aleitamento materno nas capitais brasileiras e Distrito Federal, Brasília, Ministério da Saúde; 2009.

4. Crisci P, Moura A, Alencar C. Erosão dentária em crianças ansiosas: relato de caso clínico. J Health Sci Inst. 2014;32(1):86-9.

5. Lussi A, Jaeggi T, Zero D. The role of diet in the aetiology of dental erosion. Caries research. 2004;38 Suppl 1:3444.

6. Gregg T, Mace S, West NX, Addy M. A study in vitro of the abrasive effect of the tongue on enamel and dentine softened by acid erosion. Caries research. 2004;38(6):557-60.

7. Comar LP, Salomão PMA, de Souza BM, Magalhães AC. Dental erosion: an overview on definition, prevalence, diagnosis and therapy. Brazilian Dental Science. 2013;16(1):6-17.

8. Rios D, Magalhaes AC, Honorio HM, Buzalaf MA, Lauris JR, Machado MA. The prevalence of deciduous tooth wear in six-year-old children and its relationship with potential explanatory factors. Oral health \& preventive dentistry. 2007;5(3):167-71.

9. Ushida CE, Cury JA. Estudo in situ do efeito da frequência de ingestão de Coca-Cola na erosão do esmalte-dentina e reversão pela saliva. Revista de Odontologia da Universidade de São Paulo. 1999;13(2):127-34.
10. Linnett V, Seow WK. Dental erosion in children: a literature review. Pediatric dentistry. 2001;23(1):37-43.

11. Kreulen CM, Van 't Spijker A, Rodriguez JM, Bronkhorst EM, Creugers $\mathrm{NH}$, Bartlett DW. Systematic review of the prevalence of tooth wear in children and adolescents. Caries research. 2010;44(2):151-9.

12. Milosevic A, Young PJ, Lennon MA. The prevalence of tooth wear in 14-year-old school children in $\mathrm{Li}$ verpool. Community dental health. 1994;11(2):83-6.

13. Millward A, Shaw L, Smith A. Dental erosion in four-year-old children from differing socioeconomic backgrounds. ASDC journal of dentistry for children. 1994;61(4):263-6.

14. Ministério da Saúde, Ministério do Planejamento, Orçamento e Gestão. Pesquisa de orçamentos familiares 2002-2003: primeiros resultados: Brasil e grandes regiões Rio de Janeiro: IBGE; 2004. Available from: http:// biblioteca.ibge.gov.br/visualizacao/livros/liv4472.pdf.

15. Corrêa FNP, Murakami C, Carvalho TS, Corrêa MSNP. Diagnóstico, prevenção e tratamento clínico da erosão dentária. Rev Assoc Paul Cir Dent. 2011;65(1):12-9.

16. Lussi A, Ganss C. Erosive tooth wear: Monogr Oral Sci. Basel; 2014.

17. Brusius CD. Erosão dentária em adolescentes de Porto Alegre, RS [Dissertação]: Universidade Federal do Rio Grande do Sul. Faculdade de Odontologia. Programa de Pós-Graduação em Odontologia, 2013.

18. Magalhães A, Rios D, Buzalaf M. Influência dos fluoretos na prevenção de lesões erosivas. In: Buzalaf $M$, editor. Fluoretos e saúde bucal. 2 ed. São Paulo: Santos; 2008.
REV. ODONTOL.

UNIV, CID, SÃO PAULO

$2016 ; 28(3): 262-$ 9, SET-DEZ 
19. Cury JA. Uso do flúor e controle da cárie como doença. In: Baratieri L, editor. Odontologia Restauradora: fundamentos e possibilidades. São Paulo: Santos, 2001. p. 31-68.

20. Ministério da Saúde. Guia de recomendações para o uso de fluoretos no Brasil Brasília: Ministério da Saúde; 2009. Available from: http://cfo.org. br/wp-content/uploads/2010/02/livro_ guia_fluoretos.pdf.

21. Messias DCF, Serra MC, Turssi CP. Estratégias para prevenção e controle da erosão dental. RGO Revista Gaúcha de Odontologia (On-line). 2011;59:0713.
22. Sorvari R, Meurman JH, Alakuijala P, Frank RM. Effect of fluoride varnish and solution on enamel erosion in vitro. Caries research. 1994;28(4):22732.

23. Lussi A, Megert B, Eggenberger D, Jaeggi T. Impact of different toothpastes on the prevention of erosion. Caries research. 2008;42(1):62-7.

Recebido em 02/08/2016

Aceito em 01/12/2016
FONTES CL

LUCIANO COL

FERREIRA MC

PASCHOAL MAB

ABORDAGEM DA

EROSÃO DENTÁRIA

NA CLÍNICA

ODONTOPEDIÁTRICA:

RELATO DE CASOS

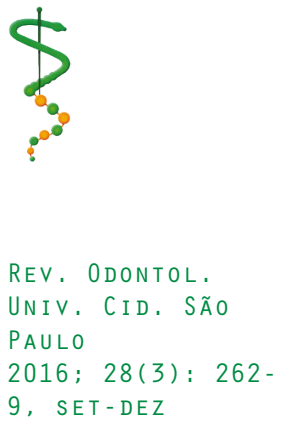

